\title{
ARTICLE
}

\section{An introduction to peer-supported open dialogue in mental healthcare}

\author{
Russell Razzaque \& Tom Stockmann
}

Russell Razzaque is a consultant psychiatrist and Associate Medical Director at NELFT NHS Foundation Trust. He is also an honorary senior lecturer at University College London, and his research interests include mindfulness, therapeutic relationships in psychiatry and open dialogue. He is a trained mindfulness teacher and also a trainer in the Academy of PeerSupported Open Dialogue. Tom Stockmann is an ST5 in general adult psychiatry at NELFT NHS

Foundation Trust. He has completed the Diploma in Peer-Supported Open Dialogue, and his research interests include reflective practice in medical education and open dialogue. Correspondence Dr Russell Razzaque, NELFT NHS Foundation Trust, Goodmayes Hospital, Barley Lane, Ifford, IG3 8XJ, UK. Email: russell.razzaque@nelft.nhs.uk

\begin{abstract}
SUMMARY
Peer-supported open dialogue is a variant of the 'open dialogue' approach that is currently practised in Finland and is being trialled in several countries around the world. The core principle of the approach is the provision of care at the social network level, by staff who have been trained in family, systems and related approaches. These staff include peer workers, who will help to enhance the democratic nature of the meetings around which care is centred, as well as enabling such meetings to occur where networks are fragmented or lacking. Certain organisational and practice features and underlying themes are key to the approach. Crucially, open dialogue is also a system of service provision. Staff trained in peersupported open dialogue from six National Health Service (NHS) trusts will launch pilot teams in 2016, as part of an intended national multicentre randomised controlled trial.
\end{abstract}

\section{LEARNING OBJECTIVES}

- Be able to describe the organisational principles of peer-supported open dialogue

- Be able to summarise the practice principles of peer-supported open dialogue

- Gain an increased understanding of the evolving role of peer-supported open dialogue in the NHS

\section{DECLARATION OF INTEREST}

None

'Open dialogue' is a holistic, person-centred model of mental healthcare pioneered in Finland that has since been taken up (to a greater or lesser degree) in a number of countries around

TABLE 1 Two-year outcomes for first-episode psychosis treated with open dialogue in Finland

\begin{tabular}{|lcc|}
\hline & $\begin{array}{c}\text { Open dialogue approach, } \\
\mathbf{n = 2 3}\end{array}$ & $\begin{array}{c}\text { Treatment as usual, } \\
\boldsymbol{n = 1 4}\end{array}$ \\
\hline Mild/no symptoms & $19(82 \%)$ & $7(50 \%)$ \\
\hline Relapse & $6(26 \%)$ & $10(71 \%)$ \\
\hline Studying, working or job seeking & $19(83 \%)$ & $4(30 \%)$ \\
\hline Antipsychotic use & $8(35 \%)$ & $14(100 \%)$ \\
\hline Mean hospital admissions, days & 14.3 & 116.9 \\
\hline
\end{tabular}

Source: Seikkula et al (2003). the world, including Scandinavian countries, Germany, Ireland, Italy (where a multi-city trial is planned) and Poland. Aspects of the approach are also being piloted in several US states. Open dialogue involves a psychologically consistent family and social network approach, in which all staff receive training in family, systems and related methodologies, and treatment is largely carried out via whole-system/network meetings that include the patient. It is a recovery-oriented model, in which the emphasis is on the mobilisation of resources within patients and their families, in order to engender a sense of agency from early on. In many respects, it is quite a different approach to much of UK service provision, in which care is essentially organised and delivered at the level of the individual. The key aspects of this difference are outlined in a paper by Olson et al (2014):

'The practice of Open Dialogue [...] has two fundamental features: (1), a community-based, integrated treatment system that engages families and social networks from the very beginning of their seeking help; and (2), a "Dialogic Practice," or distinct form of therapeutic conversation within the "treatment meeting" [...] The treatment meeting constitutes the key therapeutic context of Open Dialogue by unifying the professionals and the network into a collaborative enterprise'.

Open dialogue is now being explored as a potential future model of care by a number of National Health Service (NHS) trusts. Part of the reason for this are the promising data from Finnish non-randomised trials, which demonstrate outcomes far superior to those in the UK. For example, more than $70 \%$ of people with first-episode psychosis treated with an open dialogue approach returned to study, work or work-seeking within 2 years, despite lower rates of medication and hospital admission compared with treatment as usual (Table 1), and these outcomes were stable after 5 years (Seikkula 2006, 2011a). It should be noted that although this research looked specifically at first-episode psychosis, the evaluated open dialogue service was run in a transdiagnostic way.

Six NHS trusts are in the process of setting up pilot peer-supported open dialogue (POD) services: NELFT NHS Foundation Trust, North Essex 
Partnership University NHS Foundation Trust, Nottinghamshire Healthcare NHS Foundation Trust, Kent and Medway NHS and Social Care Partnership Trust, Avon and Wiltshire Mental Health Partnership NHS Trust and Somerset Partnership NHS Foundation Trust. These trusts plan to evaluate these services, which will potentially deepen the evidence base and ultimately spur a larger-scale take-up should the improvement in outcomes and cost reductions remain consistent. As in Finland, these are being set up as transdiagnostic services.

This article offers an introduction to POD. It begins by making a brief case for change, before giving an overview of the development of POD and then outlining its core organisational and practice aspects. It highlights the mindful nature of the model, the importance of cultivating agency on multiple levels, considerations of governance and risk, and the key role of peer workers. Finally, some of the challenges to implementing POD in the NHS are considered.

\section{The case for a social model of care}

Recent papers in the psychiatric literature have called for a new approach to mental healthcare in the UK, given the evidence of the often poor or limited outcomes achieved by current approaches (Bracken 2012) and a lack of significant progress in academic research over the past three decades (Priebe 2013). One major criticism of the prevailing approach is that it is overwhelmingly guided by a technical paradigm (Bracken 2012); this neglects the social aspect of mental health, the importance of which is strongly supported by evidence. An editorial in the British Journal of Psychiatry in 2013 speculated that the future of academic psychiatry may be social (Priebe 2013).

Key evidence is provided in the World Health Organization (WHO) International Pilot Study of Schizophrenia (WHO 1979), which unexpectedly found a markedly better overall outcome for people with schizophrenia in India and Nigeria, compared with higher-income (more 'developed') countries. This was later confirmed by the more rigorous Study on Determinants of Outcome of Severe Mental Disorder (DOSMeD), which found that 'high rates of complete clinical remission from schizophrenia were significantly more common in developing country areas than in developed countries', and that 'patients in developing countries experienced significantly longer periods of unimpaired functioning in the community'. This was despite much lower continuous use of antipsychotic medication in the 'developing' countries (Jablensky 2008).
Jablensky \& Sartorius (2008) state that:

'the sobering experience of high rates of chronic disability and dependency associated with schizophrenia in high-income countries, despite access to costly biomedical treatment, suggests that something essential to recovery is missing in the social fabric'.

There is evidence for the importance of social relationships in the cause and healing of mental disorders. For example, Giacco et al (2012) found that 'having friends is associated with more favourable clinical outcomes and a higher quality of life in mental disorders', and a Cochrane systematic review of randomised controlled trial (RCT) evidence suggests that in schizophrenia, socially based treatments such as family therapy can reduce the probability of hospital admission by around $20 \%$ and the probability of relapse by around 45\% (Pharoah 2010). Despite this evidence, models of mental healthcare based on social relationships have declined in the UK as the field has shifted towards an individualised focus (Priebe 2013).

\section{The development of POD}

The origins of the open dialogue approach lie in the 1960s in Finland (Seikkula 2015), when Yrjö Alanen and others began a long process of research and development with the aim of improving the local mental health system. Working with a largely ward-based system, they gradually integrated family perspectives over time. This led to the Finnish National Schizophrenia Project in the 1980s (discussed in Alanen 1997), which identified the following key factors for success (Alanen 1991):

- rapid early intervention

- a 'therapeutic attitude' to examination and treatment (maintaining a focus on the therapeutic process throughout rather than concentrating solely on the specific decisions made)

- ongoing, responsive and adaptive planning of tailored treatment for each patient and family

- integration of different therapeutic methods, with constant monitoring of progress.

These factors were incorporated into a method of care that was named the need-adapted treatment model (NATM) (Alanen 1997).

The family therapy field further influenced the model over the 1980s, with the incorporation of narrative, systemic and constructivist aspects. Significantly, in 1984, NATM treatment meetings began to be held in hospitals, and this model began to replace the existing local use of systemic family therapy. Difficulties encountered by NATM clinicians, such as connecting with and 
engaging families when attempting to use systemic techniques, drove the development of NATM and, eventually, open dialogue. The clinicians gradually switched from the traditional stance of a professional implementing change within a family to 'being with' the families. Another crucial change followed the influence of Tom Andersen in Norway (Andersen 1990), with the reflecting team, which in traditional family therapy would be behind a one-way mirror, being brought into the room with the family, thereby making their reflections transparent and further dissolving the professional-patient divide.

The practice of NATM eventually moved from the ward to the community, a process that was solidified in 1990 when mobile crisis intervention teams were formed by community mental health teams in local regions. This change, in the context of the other developments described here, was a critical stage in the emergence from NATM of the open dialogue approach, which was both a therapeutic model and a method of organising services. Seikkula (1994) describes the emergence from the 'rigorous evaluation of both the dialogical processes in meetings with the patients and their families, and of the outcomes in crises' of the seven core principles of open dialogue treatment (Box 1) (Keränen 1992; Seikkula 1994; Aaltonen 1997; Haarakangas 1997).

It is worth noting the interesting parallels between the NATM/open dialogue approach and the early work in the development of presentday crisis/home treatment teams. In the early days of community-focused mental health, Hoult et al (1984), in comparing community-oriented treatment with that in psychiatric hospitals, described the following important 'ingredients' for successful community treatment:

- intensive help at onset

- a willingness to actively involve both patient and relatives or carers in the management programme, as soon as possible

- consistent care by one team

- an ongoing and extensive service rather than a time-limited service

- a mobile and rapidly responsive service

- help provided in the patient's home.

Hoult also noted: 'All staff on this project quickly came to prefer this way of working. It was more satisfying and meaningful than their previous work' (Hoult 1986).

POD is developing within a context of UK crisis services and, in some respects, represents a return to key aspects of the original ethos of these services. By training staff in the therapeutic
BOX 1 Core principles of the open dialogue approach

The organisational principles of open dialogue

1 Social network perspective

2 Provision of immediate help

3 Responsibility

4 Psychological consistency

5 Flexibility and mobility

The practice-related principles of open dialogue

6 Dialogism and polyphony

7 Tolerance of uncertainty

(Seikkula 2003)

techniques involved, and increasing flexibility within the system to encourage joint working between crisis and recovery teams, the aim is to bring the core principles of the open dialogue approach, as well as the additional benefits and flexibility afforded by peer support, into acute front-line NHS services.

\section{Core organisational principles}

Open dialogue has seven core principles, of which five are organisational and two relate to practice (Seikkula 2003) (Box 1). The principles relating to the organisation of the treatment system are:

- a social network perspective

- the provision of immediate help

- responsibility

- psychological consistency throughout the care pathway

- flexibility and mobility in the provision of care.

\section{Social network perspective}

The social network perspective is fundamental to the open dialogue model. Patients' families and other key members of their social network are always invited to network meetings. Other key members may include official agencies such as Social Services and local employment agencies to support vocational rehabilitation - as well as fellow workers and any other associates or carers that may be involved. The network meeting also incorporates at least a couple of team members, and all key discussions about care take place within the network meeting. The meeting functions in a very person-centred way, which is more collaborative and less hierarchical than ordinary clinician-patient interactions. This is elaborated further under the key practice parameters outlined in the next section. 
Conversations between clinicians will also take place in front of the entire network, and all present are invited to comment on them, so that a dynamic of openness and reflection is established from the outset (Andersen 1995).

The location and composition of network meetings depends on the wishes of the patient. Often, they will be held at the patient's home. According to Finnish research, home meetings may help to prevent unnecessary hospital admissions, by rendering the family's own resources more accessible (Keränen 1992).

\section{The provision of immediate help}

Providing a rapid response, usually within 24 hours, at the point of referral is at the core of the model. The patient will be present from the start, even through the most intense phases of presentation - including psychosis - so as to create a sense of security from the outset and thus bring about a firm foundation for community and network meeting-based care.

\section{Responsibility and psychological continuity}

The first team members to be involved in the initial meeting will remain involved throughout the care pathway. This means that the same team is responsible for the treatment for as long as it takes in both out-patient and in-patient settings. Throughout treatment, the network meeting is seen as the 'sovereign' decision-making body. There will be at least two clinicians in the network meetings, who may be medical staff, depending on the nature of the case. If support for a change in medication becomes necessary, then doctors (or non-medical prescribers) can be co-opted onto the network meetings at a later stage, if there is not already a team member with the appropriate background. In addition, other modes of treatment - such as occupational therapy groups and psychotherapy - can take place between the network meetings, enabling various methods of treatment to be combined as part of an integrated process.

\section{Flexibility and mobility in the provision of care}

Flexibility around the treatment provided is vital. All conceptualisation around what is and is not appropriate or necessary is left at the door, so as to allow appropriate responses and interventions to evolve in a need-adapted way through the meetings. In psychotic crises, for example, allowing the possibility of meeting every day for up to a couple of weeks may often be necessary to generate an adequate sense of security around the crisis. Other forms of treatment and therapeutic methods are chosen depending on what best fits the patient's problems.

Additional possible interventions such as medication should, wherever possible, be discussed at several network meetings before decisions are made. This is to maintain a consistently democratic and reflective process that then facilitates the continued cultivation of a powerful sense of agency for non-clinicians when it comes to both decision-making and 'meaning formation' (see 'Cultivating agency' below) from the outset.

For the group to function in such a truly democratic and effective way, the practice of the meetings themselves will need to be guided by a set of key principles.

\section{Core principles of practice}

The practice-related principles of open dialogue form the backbone of the network meetings. These principles include dialogism and polyphony, and tolerance of uncertainty (Seikkula 2003).

\section{Dialogism and polyphony}

The term dialogism was first coined by Russian philosopher Mikhail Bakhtin in his work of literary theory, The Dialogic Imagination (Holquist 1981). The term refers to the way in which all language and thought is a process of evolution, in which every discourse/thought is a product of all the discourses/thoughts that went before it. For a dynamic to be dialogical, therefore, it must start without fixed objectives, within certain parameters, so as to allow for a free exchange that builds up layer by layer, via each contribution made, into new terrain. In addition, unlike the dialectical dynamic, there is no goal of a merging of viewpoints in order for a shared perspective to be reached. Each person can maintain their own perspective, and each perspective can hold more salience in particular circumstances - depending on the needs at the time. As a result, the group can ultimately function in a wholly pragmatic manner, enabling empowered and innovative problemsolving and decision-making, with each member having an equal right to contribute and to affect the future direction, to acquire a greater sense of agency in their own life (Haarakangas 1997).

Such a dynamic can have a therapeutic effect from the outset, enabling a sense of personal independence, as well as interdependence, to be experienced by each member of the network. As Olson et al (2014) add:

'[...] the starting point of a dialogical meeting is that the perspective of every participant is important and accepted without conditions. This means that 
the therapists refrain from conveying any notion that our clients should think or feel other than they do. Nor do we suggest that we know better than the speakers themselves what they mean by their utterances.'

Open dialogue allows each person to enter the conversation in their own way. The primary focus is on promoting dialogue (more so than promoting change in the family), and the goal of the dialogue is not agreement, but for everyone to be heard. This multiplicity of accepted voices is known as polyphony:

'The team cultivates a conversational culture that respects each voice and strives to hear all voices [...] Listening intently and compassionately as each speaker takes a turn and making space for every utterance, including those made in psychotic speech' (Seikkula 2005).

Each person in the dialogue constructs the problem using their own voice. For the clinician, listening to and responding to these voices takes precedence over interviewing techniques (Anderson 1997). Through the resulting dialogue, problems may be reconstructed and new understandings formed (Andersen 1995).

\section{Tolerance of uncertainty}

Uncertainty, on the part of both the patient and the clinician, pervades the experience of mental illness and psychological distress. The open dialogue approach explicitly acknowledges this from the outset. According to the model, however, the reflexive desire to remove the uncertainty is often the very thing that compounds it. Meetings are therefore facilitated to avoid premature conclusions or decisions about treatment (Anderson 1992). Connection to the distress being experienced is key, and this means not acting too rapidly to bring about change. If this kind of tolerance is constructed, more possibilities emerge for the family and the individual, who can then become agents of change themselves, having more robustly evolved a language to express their experience of difficult events in the intervening period. For this reason, questions are kept as openended and as relationally focused as possible, to enable the collective dialogue itself to produce a response or, alternatively, dissolve the need for action altogether.

Focusing on connection - as opposed to direction - from the outset is also a means by which safety is fostered within the meeting. Creating a safe space where everyone can be heard and respected on an ongoing basis opens up a new means by which a sense of safety can be instilled within the group. However, as Olson et al recognise, this new way of working can present a significant challenge for clinicians: 'This therapeutic position forms a basic shift for many professionals, because we are so accustomed to thinking that we should interpret the problem and come up with an intervention that counteracts the symptoms' (Olson 2014).

\section{A mindful approach}

\section{Being in the present moment}

Clinicians often approach their work with a set of templates and internal algorithms that help them make decisions about how to respond. Unfortunately, one of the consequences of this is that patients and carers can be left feeling unheard. The interaction becomes about extracting or imparting information ('doing to'), rather than 'being with' the patient and whatever is happening in the present. This moment-to-moment connectivity is a core aspect of mindfulness, and studies have shown that the ability to engage in this way has a positive effect on the therapeutic relationship (Lambert 2008; Razzaque 2015). Lambert $\&$ Simon add that mindfulness training, by potentially fostering an attitudinal change in clinicians towards greater acceptance and positive regard for self and others, represents 'an extremely promising addition to clinical training' (Lambert 2008).

A key practice in open dialogue, therefore, is to respond to the patient's utterances as they occur and keep the focus on what is happening in the here and now. According to Olson et al (2014),

'The clinician emphasizes the present moment of meeting. There are two, interrelated parts to this: (A) responding to the immediate reactions that occur in the conversation; and (B) allowing for the emotions that arise'

The focus, therefore, is wholly on the patient and those around them, and on what is happening now. As Seikkula (2011b) explains,

'Therapists are no longer interventionists with some preplanned map for the stories that clients are telling. Instead, their main focus is on how to respond to clients' utterances'.

Attention to the present moment is also a gateway through which connections can be established at a pre-verbal level. This is another way in which open dialogue is a mindful approach; all levels of presence and connection - not just the verbal are seen as vital, and cultivating an awareness of and sensitivity to them is key. Seikkula (2011b) talks of:

'moving from explicit knowledge to the implicit knowing that happens in the present moment as embodied experience, and mainly without words that is, becoming aware of what is occurring in us before we give words to it. We live in the present moment lasting only [a] few seconds. This refers 
to the micro aspects of a dialogue in the response and responsiveness of the therapist to the person before anything is put into words or described in language; that is, in being open to the other'.

As in mindfulness, the embodied connection with the other is thus believed to be as important as the verbal one: 'Therapists and clients live in a joint, embodied experience that happens before the client's experiences are formulated in words. In dialogue an intersubjective consciousness emerges' (Seikkula 2011b).

\section{Acceptance of thoughts and emotions}

Mental health professionals can often see it as their job to remove difficult thoughts and emotions. In open dialogue, however, a key skill is the ability to accept and allow whatever thoughts and emotions are happening in the present moment - as long as there is no immediate threat - to emerge and be experienced. As articulated by Olson et al (2014),

'When emotions arise such as sadness, anger, or joy, the task of therapists is to make space for their emotions in a safe way, but not give an immediate interpretation of such emotional, embodied reactions' (Olson 2014).

When this occurs, clinicians can also be 'transparent about being moved by the feelings of network members, [thus] the team members' challenge is to tolerate the intense emotional states induced in the meeting' (Seikkula 2005).

\section{Cultivating agency}

Fostering agency in the patient and their social network underpins the entire model. Agency is cultivated through the milieu that is maintained and the way decisions are made, and, as a consequence, through the way in which meaning is generated.

A key objective of working with people in this way is to enable the individual concerned to generate meaning around the experience through dialogical interaction with their social network. This more endogenous 'meaning formation', as it were, can be considered more powerful, and thus more valid and sustainable, than what could be termed exogenous meaning formation, in which outside bodies or professionals take on sole responsibility for defining the experience. By allowing for polyphony, tolerating uncertainty and connecting with the network in this way, clinicians go from being enforcers of meaning to enablers of endogenous meaning formation, therefore enhancing the sense of agency that the process itself begins to instil. In many respects, this can be seen as a core mechanism of change within the process.

\section{Risk and governance}

Risk assessments in a dialogical approach are completed and documented as in treatment as usual; however, they are compiled differently. Whereas the clinician would usually go through a checklist of questions pertaining to key elements of risk, the broader discussion in a network meeting is by definition less goal-directed. However, this wider-ranging dialogue among the many parties concerned means that issues of concern/risk - or lack thereof - arise inevitably during the course of the meeting. In this process, a far richer exchange and exploration takes place. It has been the experience of clinicians in both the UK (within the pilot teams) and abroad that by the end of a network meeting, all the items that would have been covered via direct questioning in a formal risk assessment have emerged through the dialogical interaction. Relevant details are then logged as progress notes in the appropriate formats.

A similar process has been operated and found to work effectively for other formal assessment and governance requirements such as the Care Programme Approach (CPA).

If risk arises during or around the time of the network meeting, this must be expressed in the meeting and any necessary action must be taken, whether that relates to safeguarding protocols or the Mental Health Act. This has been the practice in Finland and other countries where open dialogue or similar services operate; however, utilisation of such measures - especially detention - are reported to be required much less frequently. For this reason, whether it be in such circumstances, or for broader reasons such as prescribing medication, performing activities of daily living (ADL) assessments, engaging in supportive/recovery-oriented work and visits, or commencing one-to-one psychotherapy, the specific expertise of the individual clinician may still be called upon at any time.

Should hospital admission ultimately be required, then network meetings would still continue for the duration of the admission and after discharge. Throughout the care pathway, network meetings remain the primary decisionmaking forum when it comes to key aspects of care.

Clinicians in an open dialogue team are thus not required to abandon their area of expertise altogether. However, in a dialogical service, this expertise would normally be applied in a more discriminating, need-adapted way, within the context of a generally more democratic, less hierarchical environment. 


\section{Peer support}

Peer support is recognised as an important facilitator of individual mental health recovery (Department of Health 2008) and is currently used across a variety of mental health services (ImROC 2013; Gillard 2014; Mahlke 2014; www.hearingvoices.org). Repper \& Carter (2011) described a range of benefits for peer workers, patients and mental health services that peer worker roles offer (Box 2).

Peer support has featured in several needadapted approaches around the world that share a number of common principles with open dialogue. For example, the Parachute NYC project in New York, USA, operates along these lines, and peer workers are a core feature of the service they provide (Coe 2013; http://dcfadvertising.com/ work/parachute-nyc). Aspects of this will be replicated in the UK teams and so, in the POD model, all teams will include peer support workers. They will act as integral members of the team and have something of a dual role within it. Their first role will be to participate in network meetings alongside clinicians, utilising the principles of open dialogue. In addition to this more therapeutic role, each clinician will have their professional role (doctor, nurse, occupational therapist, etc.) which may need to be deployed, in a need-adapted way, depending on the issue at hand.

The same will apply to the peer support workers. Their expertise will be particularly called upon where the individual concerned lacks or has a

BOX 2 Benefits of peer support workers

For peer workers

- Personal discovery

- Skill development

- More likely to seek and sustain employment

- Improved financial situation

For patients

- Improved quality of life

- Increased independence

- Increased confidence

- Decreased social isolation

For mental health services

- Potential reduction in hospital admissions

- Better understanding of the challenges faced by patients

- Improved information-sharing

(Repper 2011; Gillard 2014) limited social network. The second role of the peer support worker therefore involves linking up and cultivating a local community of peers who currently receive (or have received in the past) support from local mental health services. This will be a self-help community that the peer support worker facilitates and brings forward as a resource for those who may benefit from it.

The peer support worker will also work closely with the rest of the POD team. Attendance at the main open dialogue training for staff will be key, as will attending supervision with the local team on a regular basis and receiving appropriate professional development throughout the year.

\section{Specialised training}

The POD training for NHS staff is a combination of traditional learning of the framework in order to understand the model and facilitate its operationalisation, together with experiential training to facilitate the development of the core skills. This will involve personal self-work such as mindfulness (or similar silent/contemplative practices), with the aim of developing a regular personal practice as part of an ongoing commitment to personal development.

The curriculum and learning methods are based on established open dialogue training programmes in Finland, Norway (Hopfenbeck 2015) and Massachusetts, USA.

The UK training is a joint endeavour between NELFT NHS Foundation Trust and the Norwegian University of Science and Technology. It has been organised over a 9-month period, and culminates in the award of a Foundation Diploma in PeerSupported Open Dialogue. It consists of four residential modules - each 1 week long - with an ongoing process of reading, online collaboration and facilitated dialogue among fellow students in between each of the modules.

\section{Challenges and research}

Anecdotal patient, family (Dodd 2015) and clinician experiences of POD have been extremely positive. The current empirical evidence for the effectiveness of open dialogue, however, is not sufficiently strong, nor is the approach easily transferable enough to the UK, to support the large-scale use of POD in the NHS. In addition, there are clear obstacles to the implementation of POD in the NHS, such as the organisational challenges of continuity of care in increasingly fragmented services and the cultural challenges of introducing a flattened hierarchy, increased patient and family autonomy, and peer worker involvement. 
In order to rigorously evaluate POD in the UK, a multicentre RCT is planned - with grant applications currently being prepared - to compare POD against treatment as usual, with relapse rates as the primary outcome measure. The study will centre around the provision of a POD-based model of care at the time of referral into crisis teams, and the preliminary stage to the proposed full-scale evaluation will involve the setting up of pilot teams in a number of NHS trusts to assess how best to adapt existing services to offer a POD model. The organisational challenges, in particular, will be explored at this stage, and a series of necessary adaptations for an NHS framework will likely emerge. Also central to the planned study will be the establishment of a practical POD training programme that is deliverable on a large scale, and a professional development system that is accessible to clinicians of all disciplines and sustainable in the long term.

\section{Conclusions}

The open dialogue approach is the result of an extensive, collaborative development process over several decades. Promising outcomes in Western Finland have led to the export and local modification of the approach internationally, including in New York and Berlin. POD is a further development of the approach for the NHS in the UK.

POD is a model of care that is based on strong humanistic, person-centred values. A premium is placed on establishing connections between clinicians and patients, as well as between the patient and their social network. The network meeting is seen as the crucible within which this occurs and, as a result, the clinician's role from the outset focuses more on relationships than would be the case in traditional settings. This requires a mindful, tolerant and compassionate approach to care, and it is one that will involve some personal cultivation and development on an ongoing basis. This commitment to forging a profoundly empathic connection is further enhanced by the integration into the model of peer support workers, who will contribute to a flattening of the hierarchy and, through a process of co-supervision with clinicians, enhance the patient-centred nature of the service provided.

The ultimate goal is to facilitate the emergence of a sense of agency between the patient and their social network, by allowing a dialogical milieu to form. Allowing the people most affected by the mental health concern to make sense of the experience themselves through such a dynamic becomes a key accelerator of the recovery process, and one that has the potential to create longer-term stability and ultimately promote independence from care systems and services.

There are challenges to a large-scale use of POD within the NHS, including a need for further empirical research, and significant organisational and cultural hurdles. Pilot teams are currently being set up in a number of NHS trusts to explore ways in which POD can be incorporated into a proposed multicentre study.

\section{References}

Aaltonen J, Seikkula J, Sutela M, et al (1997) Western Lapland project: a comprehensive family- and network centered community psychiatric project. In ISPS: Abstracts and Lectures 12-16 October 1997 (ed ISPS): 124. International Society for Psychological and Social Approaches to Psychosis.

Alanen Y, Lehtinen K, Aaltonen J, et al (1991) Need-adapted treatment of new schizophrenic patients: experiences and results of the Turku Project. Acta Psychiatrica Scandinavica, 83: 363-72.

Alanen Y (1997) Schizophrenia: Its Origins and Need-Adapted Treatment. Karnac.

Andersen T (1990) The reflective team. In The Reflecting Team: Dialogues and Dialogues about the Dialogues (ed T Andersen): 18-107. Bormann.

Andersen T (1995) Reflecting processes. Acts of informing and forming. In The Reflecting Team in Action: Collaborative Practice in Family Therapy (ed S Friedman). Guilford Press.

Anderson H (1997) Conversation, Language, and Possibilities. Basic Books

Anderson H, Goolishian H (1992) The client is the expert: a not-knowing approach to therapy. In Therapy as Social Construction (eds S McNamee, KJ Gergen). Sage.

Bracken P, Thomas P, Timimi S, et al (2012) Psychiatry beyond the current paradigm. British Journal of Psychiatry, 201: 430-34.

Coe S (2013) Parachute NYC: a new approach for individuals experiencing psychiatric crises. Mental Health News, 15 (2): 16 (available at https:// www.leaders4health.org/images/uploads/files/Parachute_NYC_ Article.pdf).

Department of Health (2008) A Common Purpose: Recovery in Future Mental Health Services. TSO (The Stationery Office).

Dodd C (2015) Open Dialogue: the radical new treatment having lifechanging effects on people's mental health. Independent (http://www. independent.co.uk/life-style/health-and-families/health-news/opendialogue-the-radical-new-treatment-having-life-changing-effects-onpeoples-mental-health-a6762391.html). Accessed 15 July 2016.

Giacco D, McCabe R, Kallert T, et al (2012) Friends and symptom dimensions in patients with psychosis: a pooled analysis. PLOS ONE, 7: e50119.

Gillard S, Holley J (2014) Peer workers in mental health services: literature overview. Advances in Psychiatric Treatment, 20: 286-92.

Haarakangas K (1997) [The voices in treatment meeting. A dialogical analysis of the treatment meeting conversations in family-centred psychiatric treatment process in regard to the team activity. English summary]. Jyväskylä Studies in Education, Psychology and Social Research, 130: 119-26.

Holquist M (ed) (1981) The Dialogic Imagination: Four Essays by M.M. Bakhtin (trans C Emerson, M Holquist). University of Texas Press.

Hopfenbeck M (2015) Peer-supported Open Dialogue. Context, 138 : 29-31.

Hoult J, Rosen J, Reynolds I (1984) Community orientated treatment compared to psychiatric hospital orientated treatment. Social Science \& Medicine, 18: 1005-10.
MCQ answers

$\begin{array}{lllll}1 d & 2 \text { e } & 3 b & 4 b & 5 a\end{array}$ 
Hoult J (1986) Community care of the acutely mentally ill. British Journal of Psychiatry, 149: 137-44.

ImROC (Implementing Recovery through Organisational Change) (2013) Peer support workers in mental health recovery - benefits and costs. ImROC (http://www.imroc.org/peer-support-workers-in-mental-healthrecovery-benefits-and-costs). Accessed 12 July 2016

Jablensky A, Sartorius N (2008) What did the WHO studies really find? Schizophrenia Bulletin, 34: 253-5.

Keränen J (1992) [The choice between outpatient and inpatient treatment in a family centred psychiatric treatment system. English summary]. Jyväskylä Studies in Education, Psychology and Social Research, 93. 124-9.

Lambert MJ, Simon W (2008) The therapeutic relationship: central and essential in psychotherapy outcome. In Mindfulness and the Therapeutic Relationship (eds SF Hick, T Bien): 19-33. Guilford Press.

Mahlke Cl, Krämer UM, Becker T, et al (2014) Peer support in mental health services. Current Opinion in Psychiatry, 27: 276-81.

Olson M, Seikkula J, Ziedonis D (2014) The Key Elements of Dialogic Practice in Open Dialogue: Fidelity Criteria. The University of Massachusetts Medical School (https://www.umassmed.edu/ globalassets/psychiatry/open-dialogue/keyelementsv1.109022014.pdf).

Pharoah F, Mari JJ, Rathbone J, et al (2010) Family intervention for schizophrenia. Cochrane Database of Systematic Reviews, 12: CD000088.

Priebe S, Burns T, Craig TKJ (2013) The future of academic psychiatry may be social. British Journal of Psychiatry, 202: 319-20

Razzaque R, Okoro E, Wood L (2015) Mindfulness in clinician therapeutic relationships. Mindfulness, 6: 170-4.
Repper J, Carter T (2011) A review of the literature on peer support in mental health services. Journal of Mental Health, 20: 392-411.

Seikkula J (1994) When the boundary opens: family and hospital in coevolution. Journal of Family Therapy, 16: 401-14.

Seikkula J, Aaltonen J, Rasinkangas A, et al (2003) Open dialogue approach: treatment principles and preliminary results of a two-year follow-up on first episode schizophrenia. Ethical Human Sciences and Services, 5: 163-82.

Seikkula J, Trimble D (2005) Healing elements of therapeutic conversation: dialogue as an embodiment of love. Family Process, 44: $461-75$

Seikkula J, Aaltonen J, Alakare B, et al (2006) Five-year experience of first-episode nonaffective psychosis in open-dialogue approach: treatment principles, follow-up outcomes, and two case studies. Psychotherapy Research, 16: 214-28.

Seikkula J, Alakare B, Aaltonen J (2011a) The Comprehensive OpenDialogue Approach in Western Lapland: II. Long-term stability of acute psychosis outcomes in advanced community care. Psychosis: Psychological, Social and Integrative Approaches, 3: 192-204.

Seikkula J (2011b) Becoming dialogical: psychotherapy or a way of life? Australian and New Zealand Journal of Family Therapy, 32: $179-93$

Seikkula J (2015) Open dialogues with clients and their families. Context, 138: $2-6$.

World Health Organization (1979) Schizophrenia: An International Followup Study. John Wiley \& Sons.

\section{MCOs}

Select the single best option for each question stem

1 Which of the following is not a basic principle of peer-supported open dialogue?

a Tolerance of uncertainty

b Provision of immediate help

c Flexibility and mobility

d Dialecticism

e Psychological consistency.

2 Who may be invited to a peer-supported open dialogue network meeting?

a The patient's GP

b The patient's social worker

c A staff member from a local employment agency

d The patient's carer

e All of the above.

3 Which of the following is true regarding planned peer-supported open dialogue services?

a A diagnosis of acute psychosis will be necessary for a referral to the service b Teams will aim to respond within 24 hours of a referral

c A minimum of one team member will be present at the network meetings

d No other treatment will take place outside of network meetings

e Antipsychotic medication will be entirely avoided.

4 Which of the following is false with regard to planned peer-supported open dialogue services?

a Peer support workers will be integral members of the team

b The usual risk assessment forms will not be completed

c Clinician commitment to patients' personal development may include mindfulness practice or yoga

d The Mental Health Act will be used if necessary

e The embodied connection with a patient/family will be considered to be as important as a verbal connection.
5 Regarding dialogism and polyphony, which of the following is correct?

a 'Psychotic speech' is regarded as equally valid as other voices

b If a consultant psychiatrist is present, their view always takes precedence

c Only utterances which provide useful information for a management plan will be responded to

d While not making it obvious in their questioning, clinicians should maintain an internal 'checklist' of key points to cover throughout network meetings

e The goal of the dialogue is agreement. 